ARTICLE

https://doi.org/10.1038/s41467-019-13384-y

\title{
7-Deazaguanine modifications protect phage DNA from host restriction systems
}

Geoffrey Hutinet (1* ${ }^{1 \star}$, Witold Kot $^{2}$, Liang Cui ${ }^{3}$, Roman Hillebrand ${ }^{4,12}$, Seetharamsingh Balamkundu ${ }^{3}$, Shanmugavel Gnanakalai ${ }^{3}$, Ramesh Neelakandan ${ }^{3}$, Alexander B. Carstens ${ }^{2}$, Chuan Fa Lui (1) ${ }^{5}$, Denise Tremblay ${ }^{6,7}$, Deborah Jacobs-Sera (1) ${ }^{8}$, Mandana Sassanfar ${ }^{9}$, Yan-Jiun Lee ${ }^{10}$, Peter Weigele (iD) ${ }^{10}$, Sylvain Moineau (1) 6,7, Graham F. Hatfull (1) ${ }^{8}$, Peter C. Dedon (10) ${ }^{3,4}$, Lars H. Hansen ${ }^{2}$ \& Valérie de Crécy-Lagard (1) 111*

Genome modifications are central components of the continuous arms race between viruses and their hosts. The archaeosine base $\left(\mathrm{G}^{+}\right)$, which was thought to be found only in archaeal tRNAs, was recently detected in genomic DNA of Enterobacteria phage $9 \mathrm{~g}$ and was proposed to protect phage DNA from a wide variety of restriction enzymes. In this study, we identify three additional 2'-deoxy-7-deazaguanine modifications, which are all intermediates of the same pathway, in viruses: 2'-deoxy-7-amido-7-deazaguanine (dADG), 2'-deoxy-7-cyano-7deazaguanine ( $\left.\mathrm{dPreQ}_{0}\right)$ and $2^{\prime}$-deoxy-7- aminomethyl-7-deazaguanine ( $\left.\mathrm{dPreQ}_{1}\right)$. We identify 180 phages or archaeal viruses that encode at least one of the enzymes of this pathway with an overrepresentation (60\%) of viruses potentially infecting pathogenic microbial hosts. Genetic studies with the Escherichia phage CAjan show that DpdA is essential to insert the 7deazaguanine base in phage genomic DNA and that 2'-deoxy-7-deazaguanine modifications protect phage DNA from host restriction enzymes.

\footnotetext{
${ }^{1}$ Department of Microbiology and Cell Science, University of Florida, Gainesville, FL 32611, USA. ${ }^{2}$ Department of Environmental Science, Aarhus University, Roskilde, Denmark. ${ }^{3}$ Singapore-MIT Alliance for Research and Technology, Antimicrobial Resistance Interdisciplinary Research Group, Campus for Research Excellence and Technological Enterprise, Singapore 138602, Singapore. ${ }^{4}$ Department of Biological Engineering and Center for Environmental Health Sciences, Massachusetts Institute of Technology, Cambridge, MA 02139, USA. ${ }^{5}$ School of Biological Sciences, Nanyang Technological University, 60 Nanyang Drive, Singapore 637551, Singapore. ${ }^{6}$ Département de Biochimie, Microbiologie et de Bio-informatique, Faculté des Sciences et de Génie, Université Laval, Québec City, QC G1V OA6, Canada. ${ }^{7}$ Félix d'Hérelle Reference Center for Bacterial Viruses and Groupe de Recherche en Écologie Buccale, Faculté de Médecine Dentaire, Université Laval, Québec City, QC GTV OA6, Canada. ${ }^{8}$ Pittsburgh Bacteriophage Institute and Department of Biological Sciences, University of Pittsburgh, Pittsburgh, PA 15260, USA. ${ }^{9}$ Department of Biology, Massachusetts Institute of Technology, Cambridge, MA 02139, USA. ${ }^{10}$ Research Department, New England Biolabs, Ipswich, MA 01938, USA. ${ }^{11}$ University of Florida, Genetics Institute, Gainesville, Florida 32610, USA. ${ }^{12}$ Present address: Nitto Denko Avecia, 125 Fortune Boulevard, Milford, MA 01757, USA. *email: ghutinet@ufl.edu; vcrecy@ufl.edu
} 
$\mathrm{n}$ the continuous battle between bacteria and phages, both entities are constantly evolving defenses and counterattack mechanisms ${ }^{1-5}$. To escape these defenses, phages have developed multiple strategies ${ }^{6-8}$, and one of the most widespread strategy is to modify their DNA. For example, the genomic DNA of Escherichia coli phage T4 contains the nucleobase glucosyl-hydroxymethylcytosine, which inhibits the restrictionmodification (RM) and clustered regularly interspaced short palindromic repeat (CRISPR)-CRISPR-associated (Cas) systems ${ }^{9}$. The increased availability of complete phage genome sequences has led to recent discoveries of novel complex DNA modifications, such as 2'-deoxy-5-hydroxymethyluracil derivatives in Pseudomonas phage M6, Salmonella phage Vil, and Deftia phage phi $\mathrm{W}-14^{10}$ and $2^{\prime}$-deoxyarcheosine $\left(\mathrm{dG}^{+}\right)$in Enterobacteria phage $9 \mathrm{~g}^{11}$.

Two 7-deazaguanine modifications, 2'-deoxy-7-amido-7-deazaguanosine (dADG) and the 2'-deoxyribonucleoside analog of archaeosine, which were previously thought to be present only in tRNA as queuosine $(\mathrm{Q})$ in bacteria and archaeosine $\left(\mathrm{G}^{+}\right)$in archaea, were recently discovered in bacteria and phage DNA, respectively, by combining in silico data mining and experimental validation $^{11}$. As shown in Fig. 1, 7-cyano-7-deazaguanine ( preQ $\left._{0}\right)$ is synthesized from GTP by four enzymes (FolE, QueD, QueE, QueC) and is the key intermediate in both the $\mathrm{Q}$ and $\mathrm{G}^{+}$pathways ${ }^{12-14}$. tRNA-guanine-transglycosylases (TGT in bacteria, arcTGT in archaea) are the signature enzymes in the $\mathrm{Q}$ and $\mathrm{G}^{+}$ tRNA modification pathways, as they exchange the targeted guanines with 7-deazaguanine precursors. In archaea, preQ $_{0}$ is directly incorporated into tRNA by arcTGT before being further modified by different types of amidotransferases (ArcS, GatQueC, or QueF-L) ${ }^{15-17}$. In bacteria, preQ $_{0}$ is reduced to 7aminomethyl-7-deazaguanine $\left(\right.$ preQ $\left._{1}\right)$ by $\mathrm{QueF}^{18}$ before TGT incorporates it in $\mathrm{tRNA}^{19}$, where it is further modified to $\mathrm{Q}$ in two steps ${ }^{20-22}$ (Fig. 1)

The presence of homologs of Q synthesis genes has long been reported in phage genomes ${ }^{23-26}$. However, the role of these genes in DNA modification rather than in RNA modification was only recently postulated. Indeed, TGT paralogs (now called DpdA) were found to be involved in modifying DNA in specific bacteria and phage genomes. In bacteria, the $d p d A$ gene is often located in a cluster of over ten genes that encode a RM system that inserts ADG into DNA and prevents replication of unmodified DNA $^{11,27}$. In Enterobacteria phage $9 \mathrm{~g}^{28}, d p d A$ is associated with $\mathrm{G}^{+}$synthesis, and up to $27 \%$ of the $\mathrm{dG}$ in this phage is replaced by $\mathrm{dG}^{+11,29}$. This modification is proposed to play an antirestriction role $^{28}$ because 7 -deazaguanine derivatives can block the activity of a wide variety of restriction enzymes without inhibiting the activity of the polymerases needed for phage DNA replication ${ }^{30}$.

Building on the discovery of $\mathrm{dG}^{+}$in Enterobacteria phage $9 \mathrm{~g}$, we systematically explore the genomes of other phages for potential pathways involved in 7-deazaguanine insertion in DNA and experimentally validate a subset. This work reveals a much greater diversity in the 7-deazaguanine modifications and their corresponding pathways than anticipated. Moreover, we show that 7-deazaguanine derivatives have been hijacked by phages to evade RM systems.

\section{Results}

Phage $9 \mathrm{~g}$ encodes functional preQ $_{0}$ synthesis genes. The expression of folE, queD, and queE from Enterobacteria phage $9 \mathrm{~g}$ in trans in E. coli MG1655 $\Delta$ folE, $\Delta q u e D$, and $\Delta q u e E$ strains, respectively, successfully re-established the production of $\mathrm{Q}$, demonstrating the isofunctionality of the tested pairs (Fig. 2a). This complementation was not observed when the viral gat-queC and $d p d A$ genes were expressed in $E$. coli $\Delta q u e C$ and $\Delta t g t$, respectively. The result was expected for $d p d A$, as $d p d A$ was predicted to encode an enzyme that recognizes DNA and not tRNA $^{11,31}$. This result was unexpected for gat-queC, as we had previously shown that expression of an archaeal gat-queC homolog in E. coli could lead to $\mathrm{G}^{+}$in tRNA and hence the formation of a $\mathrm{preQ}_{0}$ intermediate ${ }^{16}$.

Phage 9g Gat-QueC and DpdA insert $\mathrm{G}^{+}$DNA. As E. coli encodes the entire preQ $_{0}$ biosynthesis pathway, we predicted that the dual expression of the viral gat-queC and $d p d A$ genes in trans would lead to the insertion of 7 -deazaguanine derivatives, such as

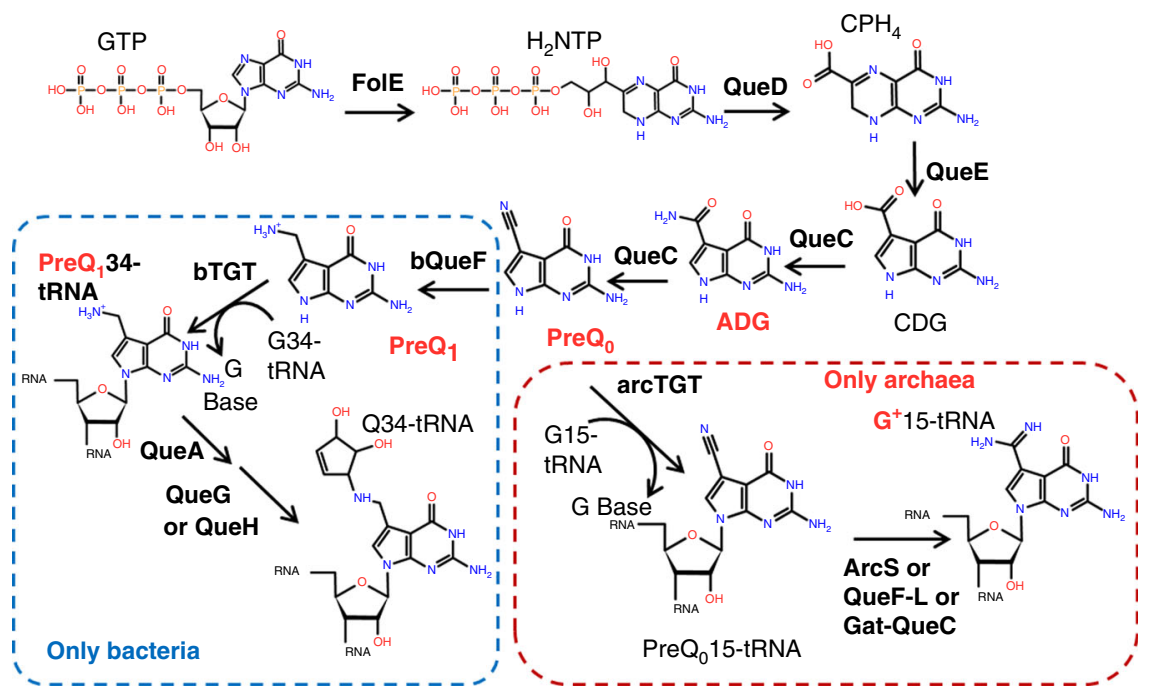

Fig. 1 Queuosine and archeosine synthesis pathways. preQ is synthesized from GTP in both bacteria and archaea through FolE, QueD, QueE, and QueC, as shown. In most bacteria, four more enzymatic steps lead to the insertion of $Q$ in tRNAs at position 34 (blue dashed square). In archaea, preQ $Q_{0}$ is transferred to position 15 of tRNA before being modified to $\mathrm{G}^{+}$(red dashed square). The bases found in phage DNA in this study are in red. Molecule abbreviations: guanosine tri-phosphate (GTP), dihydroneopterin triphosphate $\left(\mathrm{H}_{2} \mathrm{NTP}\right)$, 6-carboxy-5,6,7,8-tetrahydropterin $\left(\mathrm{CPH}_{4}\right)$, 7-carboxy-7-

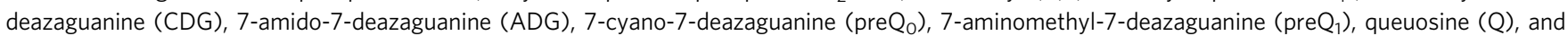
archaeaosine $\left(\mathrm{G}^{+}\right)$. 
a

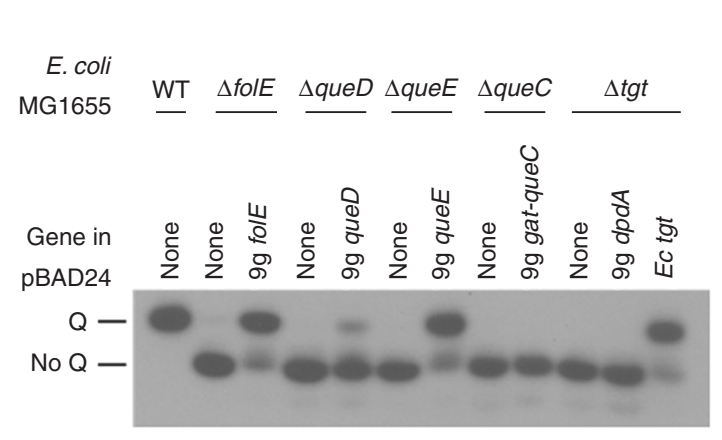

b

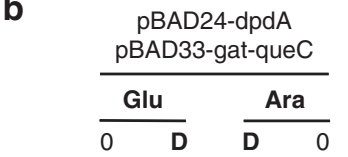

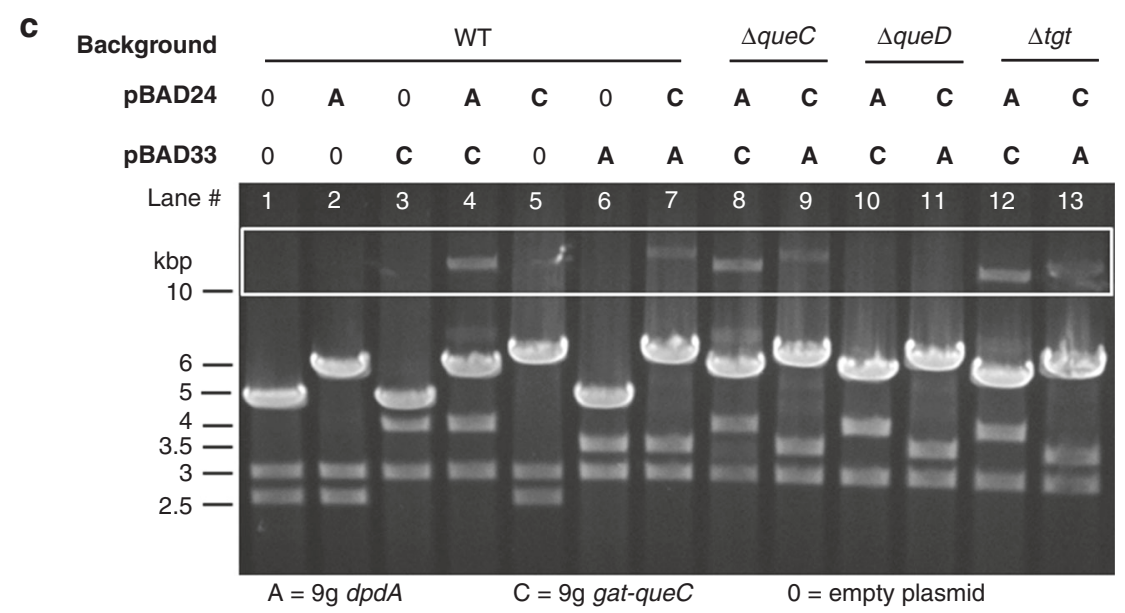

Fig. $\mathbf{2}$ In vivo activity tests for Enterobacteria phage $9 \mathrm{~g} \mathrm{dG}+$ pathway genes. Source data are provided as a Source Data file. a Northern blot of an acrylamide electromobility gel shift assay showing the tRNA-Q complementation of E. coli mutants by Enterobacteria phage $9 \mathrm{~g}$ orthologs. The WT strain modifies $\mathrm{tRNA}_{\mathrm{Asp}}$ with $\mathrm{Q}$ and is shifted in its migration (Q line), but the $E$. coli mutant strains ( $\Delta$ folE, $\Delta$ queD, $\Delta$ queE, $\Delta q u e C$, and $\Delta$ tgt) are not modified and migrate further (no Q line). In each mutant, the orthologs of Enterobacteria phage $9 \mathrm{~g}$ are expressed in trans. The complementation of $\Delta$ tgt by $E$. coli tgt is shown as a positive control of complementation. b Agarose gel of uncut (O) or EcoRI-cut (D) pGH39/pGH66 extracted from a WT strain of E. coli; expression from the plasmids was repressed in $0.4 \%$ glucose (Glu) or induced in $0.4 \%$ arabinose (Ara). White arrows indicate the undigested plasmids. c Agarose gel of EcoRI digestion of plasmids extracted from different strains of $E$. coli $(\mathrm{WT}, \Delta q u e C, \Delta q u e D, \Delta$ tgt) carrying variants of pBAD33 and pBAD24 (empty plasmid, 0 ; encoding Enterobacteria phage $9 \mathrm{~g} d p d \mathrm{~A}$, A; or encoding Enterobacteria phage $9 \mathrm{~g}$ gat-queC, C). EcoRI cuts pBAD24 once (4542-bp fragment) and pBAD33 twice (2479 and 2873-bp fragments). The resulting sizes for the digestion of pBAD24 are 5971 and 5509 bp when gat-queC or dpdA is inserted, respectively. For pBAD33, the 2873-bp fragment remains unchanged, but the 2479 -bp fragment shifts to 3911 when gat-queC is inserted and 3449 bp when $d p d A$ is inserted. When plasmids are undigested, they can be seen in the white rectangle zone.

$\mathrm{dG}^{+}$, in E. coli DNA. Because the presence of $\mathrm{dG}^{+}$confers resistance to EcoRI digestion ${ }^{29}$, we used restriction profiles as a first indication for the presence of modifications in plasmid DNA. The two phage genes were both cloned into pBAD24 and pBAD33. EcoRI cuts pBAD24 once and pBAD33 twice, as shown in the digestion profiles of plasmids extracted from E. coli cotransformed with the two empty plasmids (Fig. 2b, c, lane 1). Because the gat-queC and $d p d A$ genes of phage $9 \mathrm{~g}$ lack EcoRI sites, the restriction profiles of plasmids extracted from E. coli derivatives cotransformed with an empty plasmid and a plasmid containing one of the two genes are shifted by the insert sizes (Fig. 2c, lanes 2, 3, 5, and 6). An additional band corresponding to the uncut plasmid was observed only for plasmids extracted from strains expressing both gat-queC and $d p d A$ genes (Fig. 2c, lanes 4 and 7 , and Fig. 2b, white arrows). As a supplemental control, we digested the same combination of plasmids with PsiI (TTA^^TAA) and EcoRI (Supplementary Fig. 1). The single digestion by PsiI linearized all these plasmids, and the plasmids encoding both $d p d A$ and gat-queC of phage $9 \mathrm{~g}$ were again partially resistant to EcoRI digestion (red arrows in Supplementary Fig. 1).

Analysis of $\mathrm{dG}^{+}, \mathrm{dADG}, \mathrm{dPreQ}_{0}$, and $\mathrm{dPreQ}_{1}$ profiles by liquid chromatography-coupled triple quadrupole mass spectrometry
(LC-MS/MS, quantification results in Table 1 , mean \pm standard deviation based on two or three replicates) revealed that plasmid DNA extracted from strains expressing only $d p d A$ contained $\mathrm{dPreQ}_{0}$, with $790 \pm 8$ modifications per $10^{6}$ nucleotides; $0.316 \pm$ $0.0032 \%$ of the Gs, when expressed in pBAD24; and $84 \pm 26$ modifications per $10^{6}$ nucleotides, $0.0336 \pm 0.0104 \%$ of the Gs, when expressed in pBAD33. $\mathrm{dG}^{+}$was detected in this strain just above the detection limit as well $\left(6.5 \pm 0.5\right.$ modifications per $10^{6}$ nucleotides, $0.0026 \pm 0.0002 \%$ of the Gs). Plasmid DNA extracted from strains expressing $d p d A$ and gat-queC contained $\mathrm{dG}^{+}$, with $45,000 \pm 25,000$ modifications per $10^{6}$ nucleotides, $18 \pm 10 \%$ of the Gs, when DpdA was expressed in pBAD24 and Gat-QueC was expressed in pBAD33 and 22,750 $\pm 17,250$ modifications per $10^{6}$ nucleotides, $9.1 \pm 7 \%$ of the Gs, when reversed. $\mathrm{dPreQ}_{0}$ was also detected when gat-queC was expressed at lower levels than $d p d A, \quad\left(77 \pm 7\right.$ modifications per $10^{6}$ nucleotides, $0.0308 \pm$ $0.0028 \%$ of the Gs). No modifications were detected in strains harboring empty plasmids or when only Gat-QueC was expressed (Table 1). Taken together, these results showed that $\mathrm{dG}^{+}$but not preQ $_{0}$ confers resistance to EcoRI and that the phage $9 \mathrm{~g}$ pathway that inserts $\mathrm{dG}^{+}$in its viral DNA can be transferred to modify $E$. coli genomic DNA. 
Table 1 DNA modifications identified by mass spectrometry in the plasmids shown Fig. 2 b.

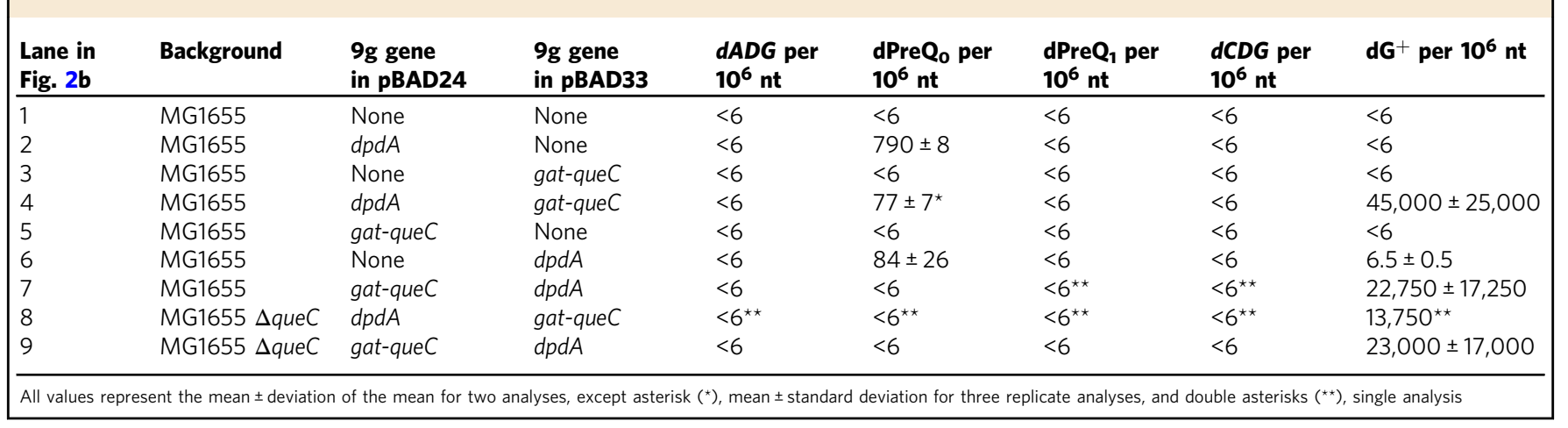

Interestingly, whereas we had failed to complement the $\mathrm{Q}^{-}$ phenotype of the E. coli $\Delta q u e C$ strain when expressing the gatqueC gene of phage $9 \mathrm{~g}$, the EcoRI resistance phenotype caused by 7-deazaguanine insertion in strains expressing both $d p d A$ and gat-queC of phage $9 \mathrm{~g}$ was still observed in a $\triangle q u e C$ background (Fig. 2c, lanes 8 and 9) but not in a $\triangle q u e D$ background (Fig. 2c, lanes 10 and 11). Furthermore, only $\mathrm{dG}^{+}$modification was observed in the DNA of the $\Delta q u e C$ strains by LC-MS/MS (Table 1), with similar amounts as in the wild type (WT; 13,750 modifications per $10^{6}$ nucleotides, $5.5 \%$ of the Gs, and $23,000 \pm$ 17,000 modifications per $10^{6}$ nucleotides, $9.2 \pm 7 \%$ of the Gs). This suggests that the Gat-QueC protein can produce preQ $_{0}$ but that it is channeled to the putative DNA-modifying enzyme DpdA and not to the tRNA-modifying pathway enzyme QueF.

Finally, we tested whether the E. coli TGT was required for DpdA activity in E. coli, as the active forms of TGT enzymes are known to be dimers ${ }^{31}$. This did not seem to be the case, as the restriction resistance phenotype was still observed in the $\Delta t g t$ background (Fig. 2c, lanes 12 and 13).

A wide variety of phages encode $\mathbf{d G}^{+}$synthesis proteins. We identified another subfamily of DpdA, renamed DpdA2, encoded by the Vibrio phage nt-1 by investigating genes flanking the preQ $_{0}$ biosynthesis gene cluster. Indeed, DpdA2 (YP_008125322) of phage nt-1 is not detected when using Enterobacteria phage $9 \mathrm{~g}$ DpdA as a query in PSI-BLAST. This DpdA2 family does not possess the conserved histidine found at position $196^{11}$. However, some similarities with members of the TGT family were detected using HHpred, with a confidence score of $100 \%$.

An in silico search for phages that could harbor 7deazaguanine derivatives in their genomic DNA revealed a total of 182 viruses deposited in GenBank that were found to encode a DpdA/DpdA2 homolog and/or at least a $\mathrm{G}^{+}$synthesis gene (Supplementary Data 1). Most of these viruses (163/182) were bacteriophages, while 16 were archaeal viruses and 3 were eukaryotic viruses. The eukaryotic viruses only encode FolE, which is most likely linked to the folate pathway ${ }^{32}$. Analyses of the presence/absence patterns of the predicted Q/G ${ }^{+}$biosynthesis genes led to a classification of these viruses into various groups and, in some cases, predicted the nature of the 7-deazaguanine base modification. It is important to note that no homologs to the proteins specifically involved in Q biosynthesis, such as QueA, QueG, or QueH (see Fig. 1), were found in the viruses analyzed.

The first group contains 25 phages and is represented by Enterobacteria phage 9g (KJ419279), Streptococcus phage Dp-1 (NC_015274), and Vibrio phage nt-1 (NC_021529) in Fig. 3. These phages encode homologs of $9 \mathrm{~g}$ DpdA or nt-1 DpdA2 as well as of FolE, QueD, QueE, and QueC. In addition, they encode homologs of one of the three amidotransferases involved in the last steps of $\mathrm{G}^{+}$synthesis: $\operatorname{ArcS}^{15}$, QueF-L ${ }^{16}$ (or QueF), or a glutamine amidotransferase (Gat) domain fused to the canonical QueC ${ }^{16}$. These phages likely modify their DNA with $\mathrm{dG}^{+}$, as does phage $9 \mathrm{~g}^{11}$. It should be noted that the discrimination between the QueF-L homologs, predicted to produce the $\mathrm{G}^{+}$base from preQ $_{0}$, and QueF homologs, predicted to produce $\mathrm{preQ}_{1}$ from pre $_{0}$, is difficult to establish based only on sequence similarity. Therefore, the phages encoding these proteins might harbor $\mathrm{dG}^{+}$ or $\mathrm{dPreQ}_{1}$ (or both). Of note, this viral group includes a Pseudomonas aeruginosa phage that was isolated; the genome of this phage was sequenced in this study, and the phage was named Pseudomonas phage Quinobequin P09 (description in Supplementary Information).

The second group includes 40 phages and is represented by $E$. coli phage CAjan (NC_028776) and Mycobacterium phage Rosebush (AY129334) in Fig. 3. These phages encode a homolog of one of the two types of DpdA and of the preQ $_{0}$ synthesis enzymes (FolE, QueD, QueE, and QueC), but they are missing an amidotransferase. As such, we predicted that these phages modify their DNA with preQ $_{0}$ or ADG, similar to the bacteria that contain the $d p d$ cluster $^{11}$. Mycobacterium phage Bipper (KU728633), which is only missing a gene coding for QueC, was added to this group even if it could be modified by the QueC substrate (7-carboxy-7-deazaguanine, see Fig. 1). The Uncultured phage clone 7AX_2 (MF417872) was also added to this group because it lacks queC, although this may be due to the incomplete genome sequence of this phage. In addition, we cannot exclude that this phage encodes an amidotransferase.

The third group is currently the largest, as it contains 76 phages, including Salmonella phage 7-11 (NC_015938) and Mycobacterium phage Orion (DQ398046), as shown in Fig. 3. These phages encode DpdA but no $\mathrm{G}^{+}$or preQ $_{0}$ biosynthesis protein homologs. At this stage, their genome modification status, if any, is difficult to predict. Phages in this group could rely on preQ $_{0}$ synthesized by the host or on the uptake of exogenous 7deazapurine precursors. Some phages do encode homologs of YhhQ, the preQ $_{0}$ transporter ${ }^{33}$, but there is no correlation with any specific group of phages. The large size of this group compared to the others might be caused by the relatively large number of Mycobacteriophages in the Virus database due to the massive phage isolation and sequencing effort of PhagesDB and the SEA-PHAGES project ${ }^{34}$.

The last group is composed of 48 phages encoding proteins of the $\mathrm{preQ}_{0} / \mathrm{G}^{+}$pathway but not DpdA. These phages could boost the production of the $\mathrm{Q}$ precursor to increase the level of $\mathrm{Q}$ in the host tRNA and increase translation efficiency ${ }^{35}$. However, it is possible that 7-deazaguanines are inserted in their DNA in a DpdA-independent pathway, as there is a recent report that the genomes of Campylobacter phages of this group are highly modified by $\mathrm{dADG}^{36}$. Similarly, the Halovirus HVTV-1 


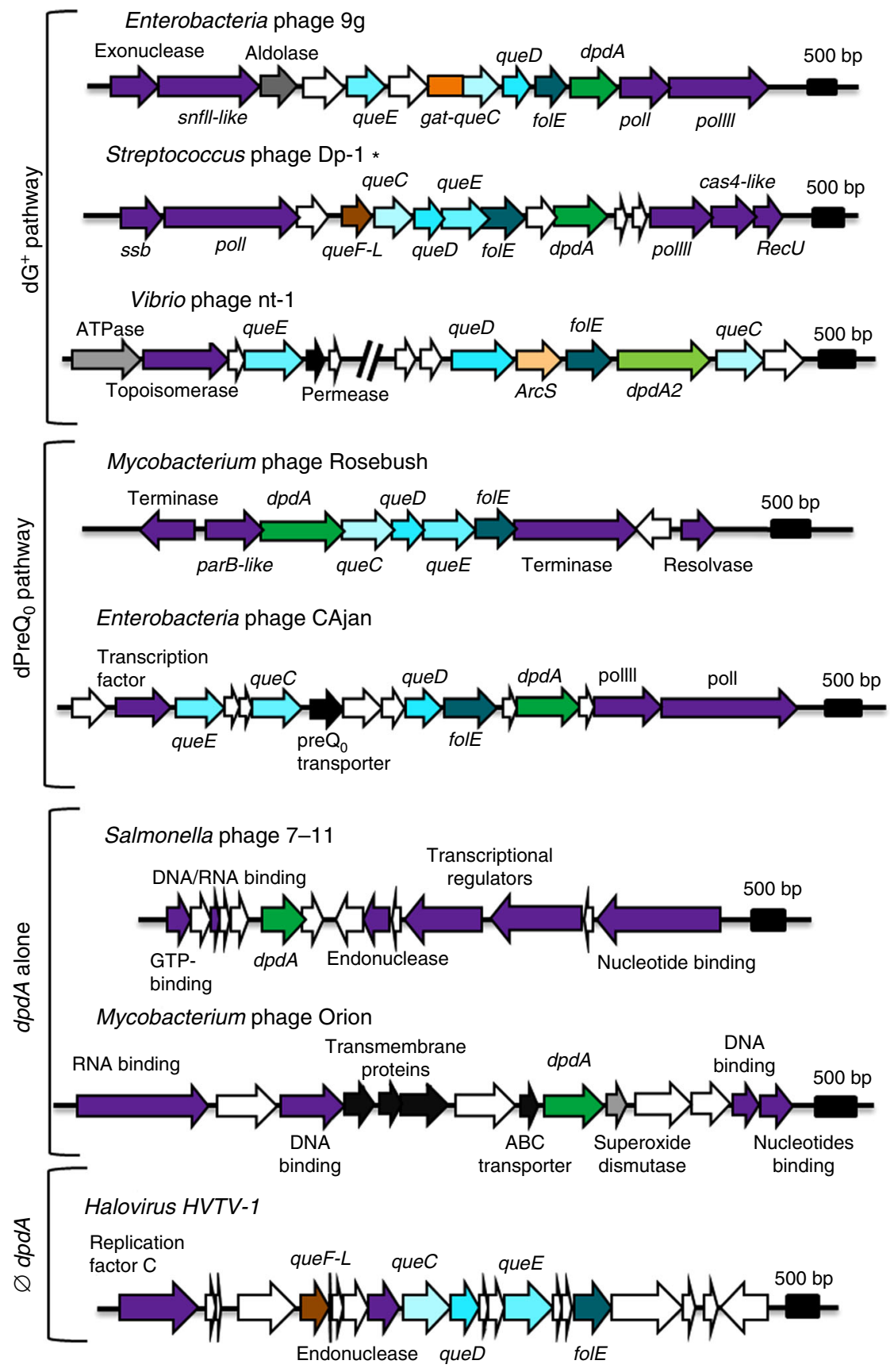

Fig. 3 Genomic context of the $d p d A$ and dG+/preQo biosynthesis pathway genes. Enterobacteria phage $9 g$, Streptococcus phage Dp-1, Vibrio phage nt-1, Mycobacterium phage Rosebush, Escherichia phage CAjan, Salmonella phage 7-11, Mycobacterium phage Orion, and Halovirus HVTV-1 are presented. The genes are colored by functions: green is DpdA, shades of blue are the biosynthetic pathway of pre $\mathrm{Q}_{0}$, shade of oranges are the genes coding for aminotransferases that synthetize $\mathrm{G}^{+}$from preQ $\mathrm{Q}_{0}$, red is QueF. In purple are the proteins involved in DNA metabolism; in black, the transmembrane proteins; in gray, all other known functions; and in white, genes coding for unknown functions. Asterisk ( $\left.{ }^{\star}\right)$ : Note that we grouped Streptococcus phage Dp-1 in the $\mathrm{dG}^{+}$biosynthesis pathway in our bioinformatics analysis, but it does not produce this modification. Source data are provided as a Source Data file.

(NC_020158), presented in Fig. 3, may have found another way to insert the modifications and should harbor either $\mathrm{dPreQ}_{1}$ or $\mathrm{dG}^{+}$, as it encodes the QueF, or QueF-like, protein.

Phages containing FolE and QueC singletons were discarded from further analysis because FolE is shared between folate and preQ $_{0}$ synthesis ${ }^{13}$, while QueC is also part of a superfamily of ATPases $^{37}$, making their precise role difficult to identify.

All the phages identified above are members of the Caudovirales order and are distributed into various families: Siphoviridae (95), Myoviridae (23), Ackermannviridae (20), and Podoviridae (3). For the Archaeal viruses, we identified 12 members of the
Ligamenvirales order and 2 of the Bicaudaviridae family (Supplementary Data 2).

Detailed analysis of phage 7-deazaguanine synthesis proteins. To evaluate the isofunctionality of the studied protein families, sequence similarity networks (SSNs) were generated. Proteins in the same cluster should share the same function ${ }^{38}$. Several of the 7-deazaguanine biosynthesis proteins are part of protein families that are known to harbor subgroups with different functions that could impede functional annotations using only PSI-BLAST 
a
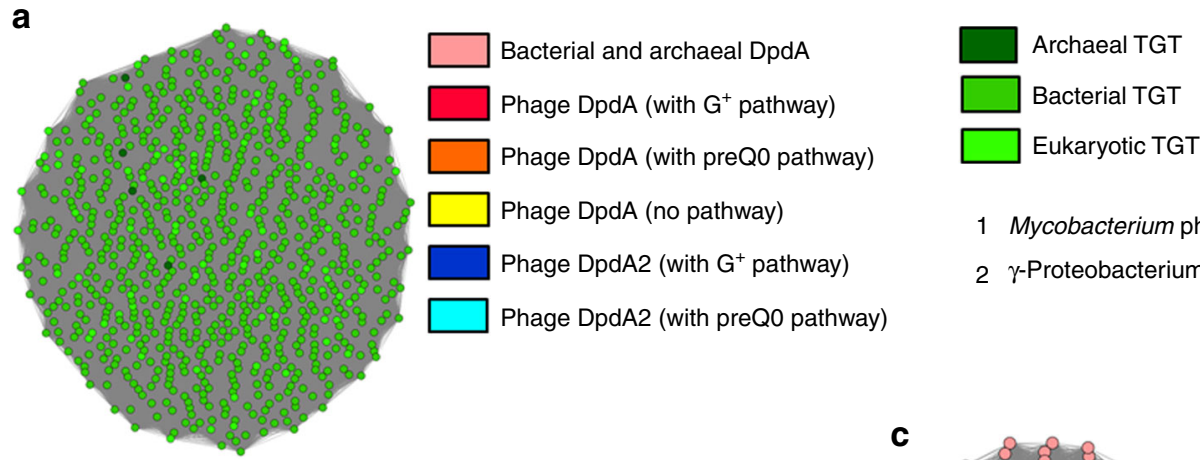

$\begin{array}{ll}1 & \text { Mycobacterium phages } \\ 2 & \gamma \text {-Proteobacterium phages }\end{array}$

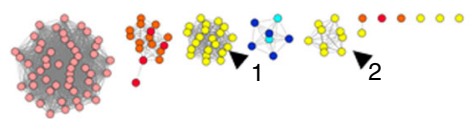

C
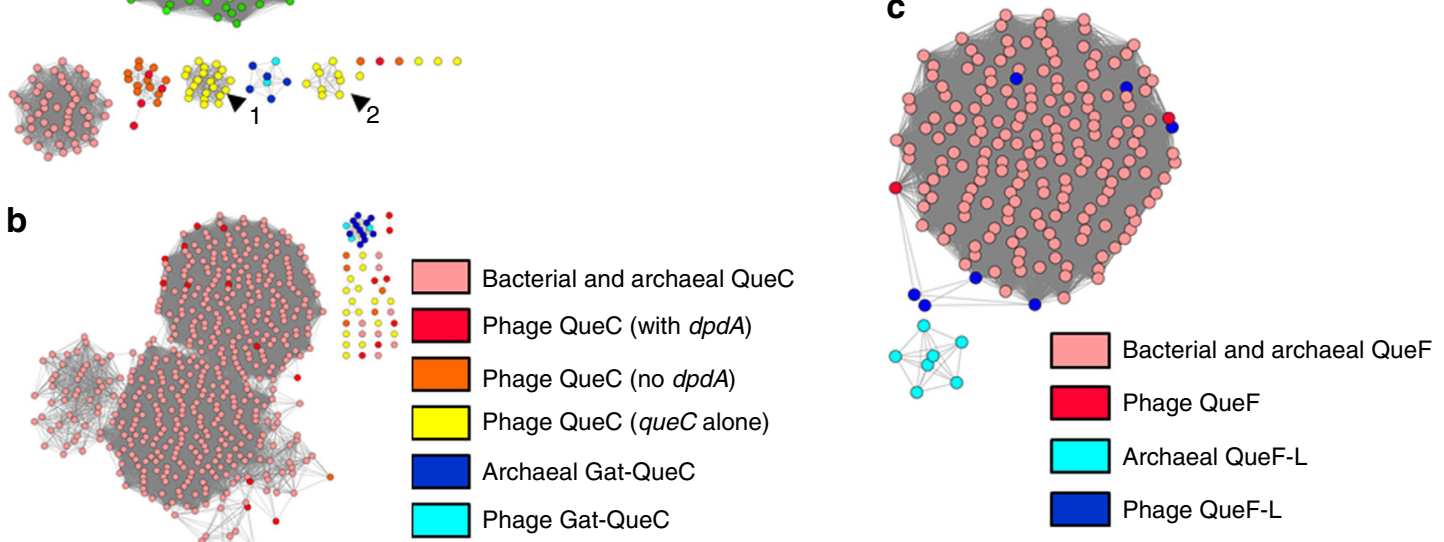

Fig. 4 Protein similarity networks. Source data are provided as a Source Data file. a DpdA/Tgt protein network, each node is a group of proteins identical at $90 \%$, and each edge presents an alignment score >15. The TGTs of archaea, bacteria, and eukaryotes are shown in dark green, green, and light green, respectively. Bacterial DpdA are shown in light red. The phage DpdA are separated depending on the gene content of phages: in red, DpdA in genomes encoding the $\mathrm{G}^{+}$pathway; in orange, the preQo pathway; in yellow, the genomes with only $d p d A$; in dark blue, DpdA2 with $\mathrm{G}^{+}$pathway; and in light blue, DpdA2 with a preQo pathway. The arrow shows clusters of nodes specific to a clade of a bacterial host ( 1 is Mycobacterium and 2 is $\gamma$-Proteobacteria). b QueC protein network, with a threshold alignment score of 44 . In light red, the QueC from bacteria; in dark red, the QueC from phages that encode a DpdA; in orange, the QueC from phages that are not encoding a DpdA; and in yellow, the QueC from phage encoding only a QueC. Gat-QueC from archaea is in dark blue and from phages is in light blue. c QueF protein network with an alignment score threshold of 10. In light red, the bacterial QueF; and in dark red, the protein identified as phage QueF. In light blue, the archaeal QueF-L; and in dark blue, the phage protein identified as QueF-L.

scores or HMM models, hence the use of SSNs to strengthen the annotation process.

As shown in Fig. 4a, phage DpdA proteins do not cluster with the TGT proteins from the three major kingdoms nor with the bacterial DpdA proteins identified previously ${ }^{11}$. Phage DpdA clearly separate in four subgroups. One contains the DpdA found in phages that encode the complete set of $\mathrm{G}^{+}$or preQ $_{0}$ synthesis proteins. The second and third groups are composed of singleton DpdA proteins, and the fourth group is composed of DpdA2 proteins. The singleton DpdAs are clustered in phages that infect the same clade of bacteria (Mycobacterium and $\gamma$-Proteobacteria). This could be a sign of a rapid divergence of this protein subfamily, and more studies will be required to determine whether this subset of DpdA proteins has functionally diverged.

Most phage QueC proteins do not cluster with bacterial QueC proteins when the BLAST threshold score is sufficient to separate QueC from the Gat-QueC groups (Fig. 4b). However, when a lower threshold score is used, the QueC and Gat-QueC proteins can be connected (Supplementary Fig. 2A). This is not the case for the QueC proteins encoded as singletons in phages, such as Bacillus phage SP-15 and Salmonella phage SFP10 (Supplementary Data 1), suggesting that even though the proteins were identified as QueC by HHpred, they may be part of a functionally unrelated subgroup of the N-type ATP pyrophosphatases superfamily ${ }^{37}$. Finally, phage and archaeal Gat-QueC proteins form a single cluster, strengthening their functional association.

HHpred predicted that the QueF family proteins encoded by phages are, for most of them, closer to the archaeal QueF-L proteins than to the bacterial QueF proteins (see Supplementary
Data 1). However, they clustered with bacterial QueF proteins in the SSNs (Fig. 4c). Further experimental studies are required to determine whether the phage QueF proteins are nitrile reductases or amidotransferases (Fig. 1).

SNNs for the FolE, QueD, QueE, and ArcS families are shown in Supplementary Fig. 2B-E. The phage proteins cluster nicely with their bacterial and archaeal homologs, reinforcing the initial functional annotations.

The host may participate in phage DNA modification. To study the interaction between phages containing 7-deazaguaninerelated genes and their bacterial hosts, we gathered metadata on the hosts and their habitat using RefSeq ${ }^{39}$ and the Globi database $^{40}$ and analyzed the distribution of $\mathrm{Q}, \mathrm{G}^{+}$, and $\mathrm{dADG}$ synthesis genes in these organisms (see Supplementary Data 2 and 3). Interestingly, 106 of the collected phages ( $60 \%)$ infect a host strain that is the model for a known bacterial pathogen (Supplementary Data 2), where only $\sim 9 \%$ of all the doublestranded DNA (dsDNA) viruses from the Virus-Host database ${ }^{41}$ infect a strain related to pathogens (data not shown), making our sample six to seven times more enriched compared to a random sampling. No clear environment was found for the archaeal hosts.

All phage hosts predicted to modify their DNA with $\mathrm{G}^{+}$ possess the pathway to produce $\mathrm{Q}$ in tRNA. Curiously, the hosts of phages coding for a QueF-L and a 9g DpdA homolog do not encode the preQ $Q_{0}$ biosynthetic pathway (QueDEC, see Fig. 1) but encode the specific preQ $_{0}$ transporter $\mathrm{YhhQ}^{33}$ and the rest of the $Q$ pathway (QueFAG and TGT, Fig. 1). Conversely, all the hosts of the DpdA2-encoding phages encode the full Q pathway. 
Table 2 DNA modifications identified by mass spectrometry in the different phages.

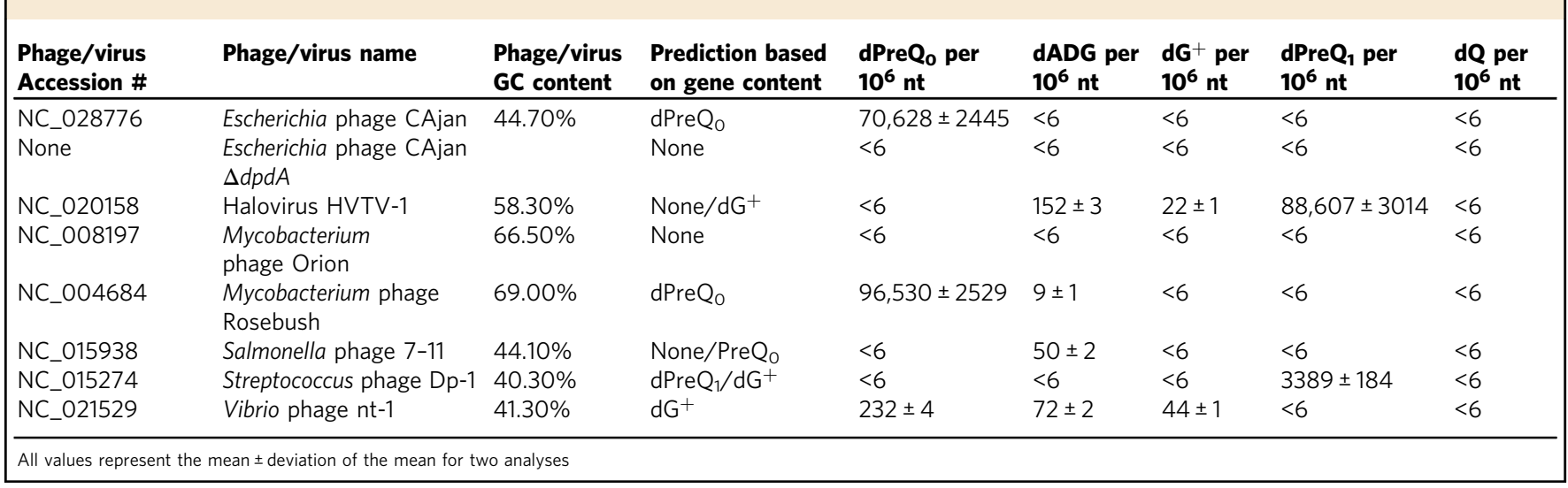

There is no clear pattern for the bacterial hosts of phages encoding both DpdA and the whole preQ ${ }_{0}$ pathway. Most of them encode the full Q pathway enzymes except for Streptococcus pneumoniae, which lacks the preQ $_{0}$ pathway genes; Rhodococcus erythropolis, which encodes only TGT; and Mycobacteria, which possess none of these genes.

The hosts of the phages encoding only DpdA also encode the full set of $\mathrm{Q}$ synthesis enzymes except the Clostridium species, which lack the preQ $_{0}$ pathway genes, and the Mycobacterium genus, which possesses none of these genes. Sulfolobi were not referenced in PubSEED ${ }^{42}$, but by performing a BLASTp search with default parameters and the genes listed in Supplementary Table 1 as queries, we identified all $\mathrm{G}^{+}$pathway genes (Supplementary Table 2). Hence, the 7-deazaguanine intermediates produced by these hosts, Clostridium and Mycobacterium excluded, might be used by phages that lack the biosynthesis proteins to produce a 7-deazaguanine precursor.

Finally, the hosts of the phages that do not encode a DpdA homolog but encode the preQ $_{0}$ pathway proteins all encode the full Q synthesis pathway.

A few bacterial hosts, such as 46 different strains of $E$. coli, Haloarcula vallismortis, and Vibrio harveyi 1DA3, also harbor homologs of the bacterial DpdA, which are known to modify bacterial DNA by either $\mathrm{dPreQ}_{0}$ or $\mathrm{dADG}^{11}$.

Different 7-deazaguanine modifications in distinct phages. To test our predictions on the nature of phage DNA modifications, a set of phages from each group were selected (Fig. 3), and their genomic DNAs were extracted for mass spectrometric analysis (Table 2, mean \pm standard deviation based on two replicates). No 2 -deoxyqueuosine (dQ) was found in any of the tested samples, correlating with the fact that no phage or virus encodes the specific protein for Q synthesis (QueAGH).

Phages of the first group encoding both a DpdA and one of the amidotransferase homologs were analyzed. Streptococcus phage Dp-1 DNA, encoding a QueF-L, contained a large amount of $\mathrm{dPreQ}_{1}\left(3389 \pm 184\right.$ modifications per $10^{6}$ nucleotides, $\sim 1.7 \pm$ $0.09 \%$ of the Gs) but no $\mathrm{dG}^{+}$, which would mean that the QueF-L of this phage would actually be functionally closer to bacterial QueF than archaeal QueF-L, as predicted by the SSN clustering (Supplementary Fig. 2). Vibrio phage nt-1, encoding an ArcS, was shown to harbor not only $\mathrm{dG}^{+}\left(44 \pm 1\right.$ modifications per $10^{6}$ nucleotides, $\sim 0.02 \pm 0.0005 \%$ of the Gs) but also $\mathrm{dPreQ}_{0}$ and dADG $\left(232 \pm 4\right.$ modifications per $10^{6}$ nucleotides, $\sim 0.11 \pm 0.002 \%$ of the Gs, and $72 \pm 2$ modifications per $10^{6}$ nucleotides, $\sim 0.035 \pm$ $0.001 \%$ of the Gs, respectively). This result might indicate that nt$1 \mathrm{DpdA}$ is more promiscuous and could insert all intermediates of the pathway.
Then we investigated phages of the second group that encode both a DpdA and the four proteins of the $\mathrm{preQ}_{0}$ biosynthesis pathway but no amidotransferase homolog. Mycobacterium phage Rosebush was found to harbor PPreQ $_{0}$ in its DNA $\left(96,530 \pm 2529\right.$ modifications per $10^{6}$ nucleotides, $28 \pm 1 \%$ of the Gs), as does Escherichia phage CAjan (70,628 \pm 2445 modifications per $10^{6}$ nucleotides, $\sim 32 \pm 1 \%$ of the Gs). However, Mycobacterium phage Rosebush was also found to harbor a negligible amount of dADG $\left(9 \pm 1\right.$ modifications per $10^{6}$ nucleotides, $\sim 0.003 \pm 0.0003 \%$ of the Gs).

The genomic DNA of Salmonella phage 7-11 and Mycobacterium phage Orion from the third group of phages, which only encode a DpdA, were also analyzed by LC-MS/MS. Mycobacterium phage Orion lacked any 7-deazaguanine modifications in its DNA. This result was expected, as none of the phage nor the host encode for the preQ $_{0}$ biosynthesis pathway (Mycobacterium smegmatis, seeSupplementary Data 3). However, Salmonella phage 7-11 was unexpectedly modified by dADG $(50 \pm 2$ modifications per $10^{6}$ nucleotides, $\sim 0.02 \pm 0.0009 \%$ of the Gs), suggesting that the phage encoded a protein responsible for the oxidation of preQ $_{0}$.

Finally, Halovirus HVTV-1, which encodes the four proteins of the preQ $_{0}$ biosynthesis pathway and a QueF-L homolog but no DpdA, contained mainly $\mathrm{dPreQ}_{1}(88,607 \pm 3014$ modifications per $10^{6}$ nucleotides, $\sim 30 \pm 1 \%$ of the Gs) but also relatively small amounts of $\mathrm{dADG}$ and $\mathrm{dG}^{+}(152 \pm 3$ modifications per $10^{6}$ nucleotides, $\sim 0.05 \pm 0.001 \%$ of the Gs, and $22 \pm 1$ modifications per $10^{6}$ nucleotides, $\sim 0.008 \pm 0.0003 \%$ of the Gs, respectively). As its host, $H$. vallismortis harbors a DpdA homolog, and it is possible that the host DpdA inserts preQ ${ }_{0}$ in Halovirus HVTV-1 DNA before it is further modified to $\mathrm{dPreQ}_{1}$ or $\mathrm{dG}^{+}$by the viral QueF-L or to $\mathrm{dADG}$ by another unidentified protein.

$\boldsymbol{d} \boldsymbol{d} \boldsymbol{d A}$ is essential for DNA modification. To evaluate the role of the 7-deazaguanine modifications in phages, we used the Escherichia phage CAjan as a genetic model. CAjan is a virulent phage belonging to the Seuratvirus genus of the Siphoviridae family with many similarities with Enterobacteria phage 9g, particularly within the 7 -deazaguanine modification pathway ${ }^{43}$. Using the CRISPR-Cas9 genome editing technology ${ }^{44}$, we generated a CAjan derivative with an inactive allele of the $d p d A$ gene (Supplementary Fig. 3A). The presence of this allele was confirmed by PCR and sequencing (Supplementary Fig. 3B). The LC-MS/MS analysis of the DNA of the mutated phage showed a complete lack of 7-deazaguanine modifications (Table 2). 
a $9 \mathrm{~g}$ WT
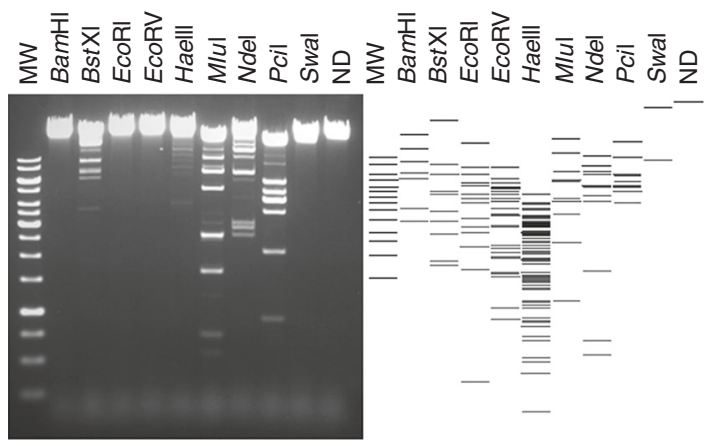

C CAjan WT

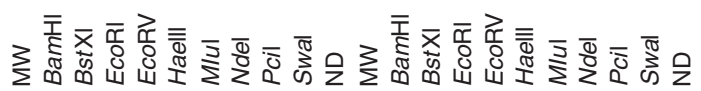

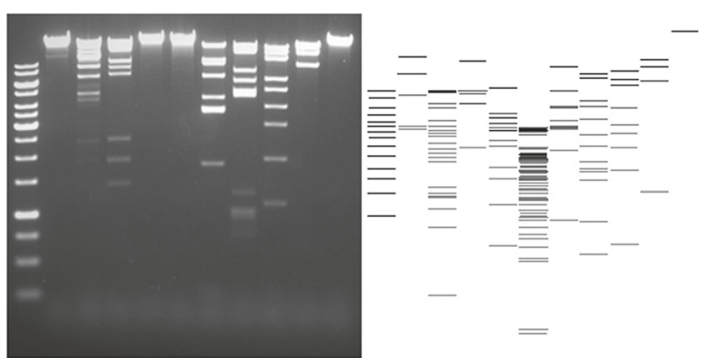

e HVTV-1

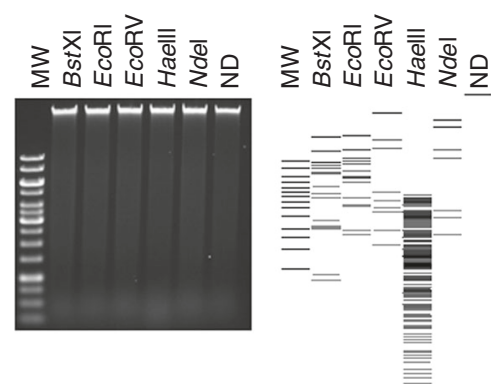

b

Rosebush WT

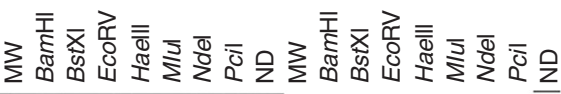

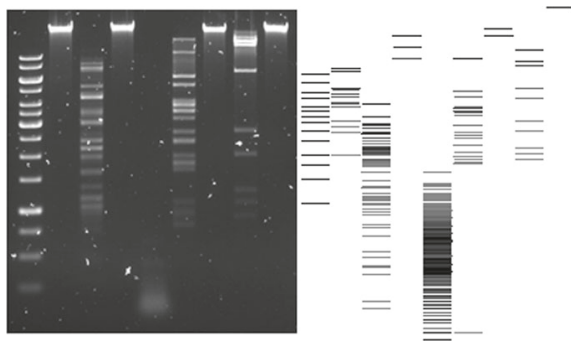

d

CAjan $\triangle \mathrm{dpdA}$

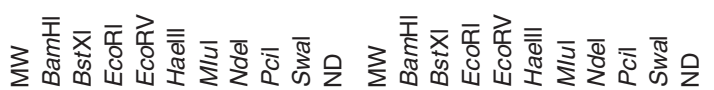

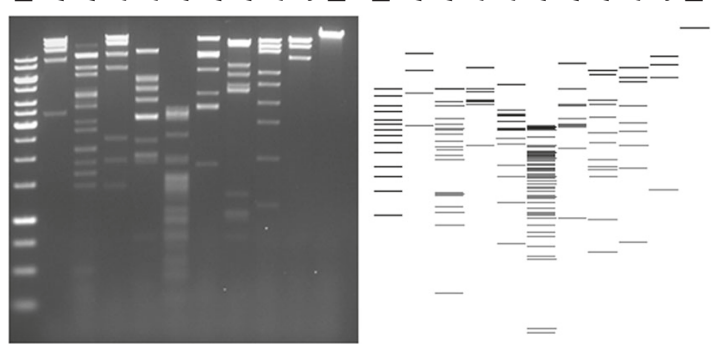

Fig. 5 Restriction patterns for phage genomic DNA. Different restriction enzymes were used on the DNA of Enterobacteria phage 9g (a), Mycobacterium phage Rosebush (b), Escherichia phage CAjan WT (c), Escherichia phage CAjan $\Delta d p d A$ (d), and Halovirus HVTV-1 (e). On the side of each gel is the representation of the expected restriction pattern. Source data are provided as a Source Data file.

The DNA modifications protect DNA from restriction enzymes. The different modifications present in the phages analyzed above may lead to distinct resistance patterns to host defense mechanisms, such as RM systems. To test this hypothesis, phage DNA preparations were digested with a set of restriction enzymes that had been shown to be totally or partially inactivated in the presence of the $\mathrm{dG}^{+}$modification ${ }^{29}$. As a control, we reproduced the results published with Enterobacteria phage $9 \mathrm{~g}$ DNA (Fig. 5a); no digestion was observed with BamHI, EcoRI, EcoRV, and SwaI, while it was partially restricted with BstXI, HaeIII, MluI, NdeI, and PciI.

Mycobacterium phage Rosebush DNA that carries preQ $_{0}$ showed a slightly different pattern of resistance. The restriction profiles for BamHI, BstXI, and EcoRV were identical to those of Enterobacteria phage 9g. However, Rosebush DNA was fully sensitive to HaeIII, MluI, and PciI and resisted NdeI degradation (Fig. 5b). EcoRI and SwaI could not be tested because the corresponding sites are absent in the Mycobacterium phage Rosebush genome.
Though Escherichia phage CAjan DNA carries the same modification as Mycobacterium phage Rosebush DNA, differences in the restriction patterns were observed (Fig. 5c). Indeed, while EcoRI and SwaI fully digested this DNA preparation, BamHI digested it only partially, and HaeIII did not cut at all. These differences could be explained by the additional small amount of dADG present in Mycobacterium phage Rosebush DNA, by the differences in modification density potentially affecting accessibility to the restriction sites, or by the presence of another undetected modification. In comparison, the $\Delta d p d A$ mutant of CAjan, lacking any modifications, was fully digested by all the tested restriction enzymes (Fig. 5d), formally linking the presence of the $d p d A$ gene and the $\mathrm{dG}^{+}$modification to the restriction resistance phenotype.

Last but not least, Halovirus HVTV-1 DNA that carries mainly $\mathrm{dPreQ}_{1}$ was found to resist restriction by all enzymes tested, even those that lack guanine in the recognition site (Fig. 5e and Supplementary Fig. 4). It is possible that this virus has other modifications that help resist restriction and, if not $\mathrm{dPreQ}_{1}$, is the 


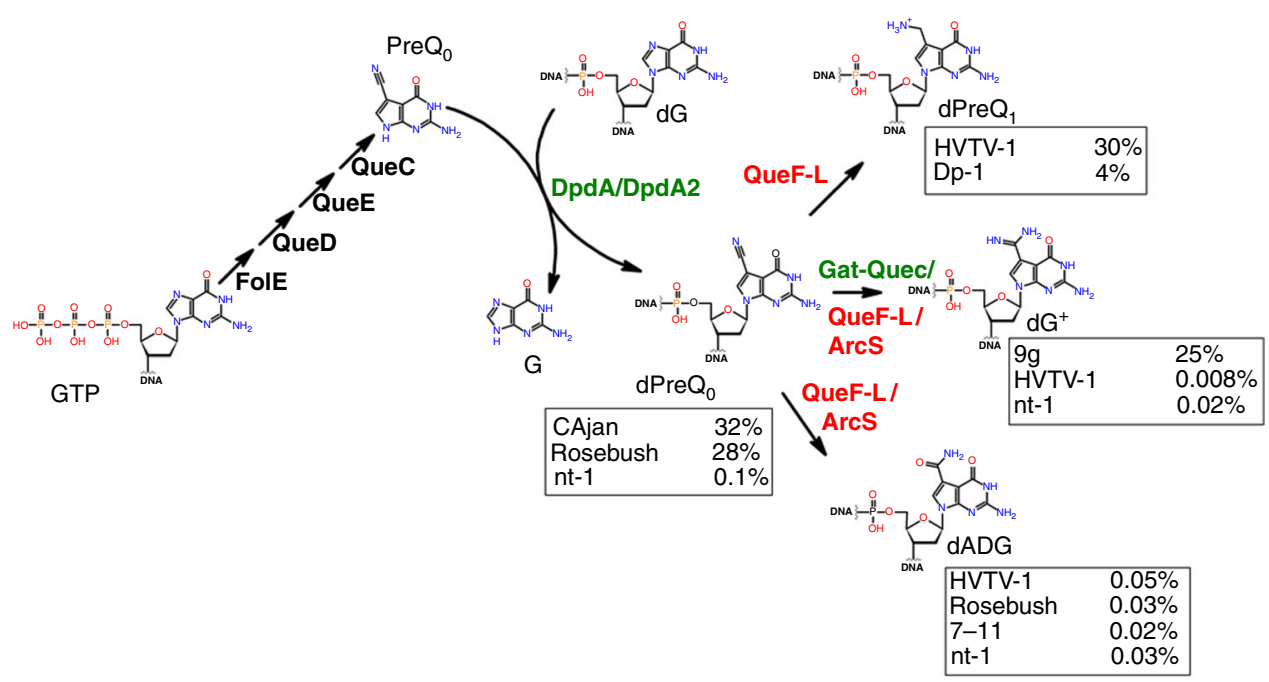

Fig. 6 Proposed synthesis pathway for the 2'-deoxy-7-deazaguanine modifications identified in this study. The percentages of modifications identified for each phage are shown in boxes next to the modification of interest. Protein names in green are the reactions identified in this study. In red are the proposed reactions. Molecule abbreviations: guanosine tri-phosphate (GTP), 7-cyano-7-deazaguanine (preQo), 2'-deoxy-7-cyano-7-deazaguanosine (dPreQo), guanine (G), 2'-deoxyguanosine (dG), 2'-deoxy-7-aminomethyl-7-deazaguanosine ( $\mathrm{dPre}_{1}$ ), 2'-deoxy-7-amido-7-deazaguanosine (dADG), and 2'deoxyarchaeaosine $\left(\mathrm{dG}^{+}\right)$.

best modification for protection from restriction enzymes identified in this study.

\section{Discussion}

In a previous study ${ }^{11}$, we identified two 7 -deazaguanine modifications in DNA: dADG in bacteria and $\mathrm{dG}^{+}$in phages. Here we added two modifications, $\mathrm{dPreQ}_{1}$ and $\mathrm{dPreQ}_{0}$, both found in phages. Similar to the result of Szymanski's group on Campylobacter phages ${ }^{36}$, we also detected dADG in phage genomes. We identified the genes involved in the synthesis of these different modifications. FolE, QueD, and QueE from Enterobacteria phage $9 \mathrm{~g}$ were shown to functionally replace their $E$. coli orthologs (Fig. 2a), and their clustering in SSNs (Supplementary Fig. 2) leaves no doubt on the isofunctionality of these families. No individual phage QueC was tested, but the strong clustering of bacterial, archaeal, and phage QueC proteins in SSNs also point to identical functions. One exception may be the singleton encoded QueC-like protein, found in Escherichia phage ECML-4 (YP_009101458 in NC_025446) or Mycobacterium phage Muddy (YP_008408902 in NC_022054), which is likely a member of another subfamily of the N-type ATP pyrophosphatases superfamily ${ }^{38}$.

Most 7-deazaguanine-containing phage genomes also harbor a gene coding for a DpdA homolog. As with its bacterial homolog 27 , the phage DpdA introduces PreQ ${ }_{0}$ in DNA (Fig. 2c, Table 1), most likely through a base exchange mechanism similar to its TGT homolog 31 . DpdA2 proteins appear to share this function, as the Vibrio phage nt-1 genome contains $\mathrm{dPreQ}_{0}$ (Table 2 and Fig. 3). However, not all phages/viruses containing 7-deazaguanines encode DpdA proteins, as observed with Halovirus HVTV-1 (Table 2 and Fig. 3). It is possible that, in the case of HVTV-1, the host DpdA is responsible for the presence of modifications in its genome (EMA11768 in AOLQ01000002). Nevertheless, a DpdA is not always present in the host, and there could be some cases where the phages encode a machinery to synthesize a modified dGTP that is used by DNA polymerase, as proposed for Campylobacter phages $^{36}$. Finally, one cannot rule out that some phages may harbor undetected 2'-deoxyribosyltransferases.

The combination of comparative genomic analyses and experimental validations has allowed pathways for the insertion of $\mathrm{dPreQ}_{0}, \mathrm{dPreQ}_{1}$, and $\mathrm{dG}^{+}$in phage genomes to be predicted
(Fig. 6). The presence of the minimal set of FolE, QueD, QueE, QueC, and DpdA proteins leads to the insertion of $\mathrm{dPreQ}_{0}$, as observed in Mycobacterium phage Rosebush and Escherichia phage CAjan genomes (Table 2 and Fig. 3). The replacement of QueC by Gat-QueC leads to the introduction of $\mathrm{dG}^{+}$(Fig. 2c, Table 1 and previous study ${ }^{11}$ ). However, it is not known whether Gat-QueC converts preQ $_{0}$ into $\mathrm{G}^{+}$before or after it is inserted into DNA. The function of ArcS homologs in phages/viruses is less clear. Indeed, Vibrio phage nt-1 encodes an ArcS homolog, and its DNA contains mainly $\mathrm{dPreQ}_{0}$ but also $\mathrm{dG}^{+}$and $\mathrm{dADG}$ (Table 2 and Fig. 3). ArcS was the first $\mathrm{G}^{+}$synthase identified in archaea $^{15}$. Based on the phage and archaeal ArcS cluster in the SNNs (Supplementary Fig. 2), it is possible that some phage ArcS protein evolved to perform not only an amidotransferase reaction, such as the archaeal $\mathrm{ArcS}^{15}$, but also an amidohydrolase reaction, such as the bacterial $\mathrm{DpdC}^{27}$. Further biochemical characterization will be required to explore these hypotheses. One cannot exclude the possibility that the small amount of dADG detected in Vibrio phage nt-1, Halovirus HVTV-1, Mycobacterium phage Rosebush, and Escherichia phage CAjan could be the result of the natural oxidation of $\mathrm{dPreQ}_{0} 45$.

The discrepancy observed between the SSNs and HHpred predictions for the QueF/QueF-L homologs was resolved by analyzing Streptococcus phage Dp-1 and Halovirus HVTV-1 DNA. HHpred analysis predicted that a homolog of the archaeal QueF-L, which synthesizes $\mathrm{G}^{+}$-tRNA from the preQ $_{0}$-tRNA ${ }^{46}$, was encoded by these phages, whereas the SSN analysis predicted that this same protein was part of a group of bacterial QueF proteins (Fig. 4) that synthesize preQ $_{1}$ from the free preQ $\mathrm{Q}_{0}$ base $^{18}$. We found that Streptococcus phage Dp-1 and Halovirus HVTV-1 were modified by $\mathrm{dPreQ}_{1}$, confirming the SSN prediction. However, it is unclear whether the reduction occurs on free preQ $Q_{0}$, similar to the bacterial QueF proteins ${ }^{18}$, and then the free base preQ $_{1}$ is inserted by DpdA or if the phage QueF is able to modify the DNA-bound $\mathrm{dPreQ}_{0}$, as does the archaeal QueF-L with tRNA $^{46}$. However, Halovirus HVTV-1 contains mainly $\mathrm{dPreQ}_{1}$ but also a small amount of $\mathrm{dADG}$ and $\mathrm{dG}^{+}$. It is possible that the QueF-L transitions between its function as an amidohydrolase to an amidotransferase, but one cannot rule out that the host ArcS could catalyze the reaction, although the PUA domain specific for tRNA binding makes it highly unlikely ${ }^{15}$. 
From a biological perspective, 7-deazaguanine modifications seem to dramatically decrease the susceptibility of phage genomes to host RM systems. RM systems are one of the major defense systems for bacteria to prevent invasion by foreign $\mathrm{DNA}^{5}$. Phages evolved to escape these RM systems by different methods, including modification of their genomic $\mathrm{DNA}^{9,11,47,48}$. It was previously observed that the genome of Enterobacteria phage $9 \mathrm{~g}$ contains $\mathrm{dG}^{+11}$ and is fully or partially resistant to a wide variety of restriction enzymes ${ }^{29}$. In this study, we directly linked the presence of the modification to the restriction resistance phenotype. Escherichia phage CAjan with mutations in $d p d A$ no longer contains $\mathrm{dPreQ}_{0}$ modifications (Table 2) and is sensitive to all the restriction enzymes tested (Fig. 5). In addition, all 7deazaguanine-modified DNA preparations tested were protected to various degrees from digestion by restriction enzymes. We also observed that introducing $\mathrm{dG}^{+}$modifications in the E. coli genome protected against cleavage by EcoRI (Fig. 2). These modifications might also block other DNA-binding proteins that require the nitrogen moiety at position 7 of the guanine to recognize their substrates, the most critical being sigma and transcription factors. However, phages only use the housekeeping sigma factor ${ }^{49}$, which has an AT-rich recognition sequence ${ }^{50}$, and encode their own transcription factors ${ }^{51}$.

Finally, the distribution of these modifications among phages seems to correlate with their host range, namely, bacterial pathogenic species. Interestingly, this was also observed in bacteria, where many pathogens harbor dADG modifications ${ }^{11}$. Although it is not clear how 7-deazaguanine modifications are spread through phage isolates, these modifications might give a selective advantage to pathogenic species. These 7-deazaguaninemodified phages are also most likely more adapted to propagate in hosts with modified DNA. We can only speculate on how bacteria evolve to counteract this specific anti-restriction mechanism. As we were successful in deleting the $d p d A$ gene from Escherichia phage CAjan using a CRISPR-Cas9 technique (see "Methods"), we know that these modifications do not provide resistance against the type II CRISPR-Cas system ${ }^{4}$. However, as the adaptive system of CRISPR-Cas recognizes the nitrogen in position 7 of the guanines in the $\mathrm{PAM}^{52}$, it is possible that these phages escape degradation by CRISPR-Cas by preventing the adaptation system from binding to its target DNA. One could also imagine that other means of defense, described in recent reviews ${ }^{2,3}$, provide an efficient protection mechanism against these phages or that some bacteria evolved means of defense yet to be discovered.

\section{Methods}

Strains, phages, plasmids, and oligonucleotides. The bacterial strains used in this study are listed in Supplementary Data 4. Phages are listed in Supplementary Data 5. Plasmids are listed in Supplementary Table 3, and plasmid constructions are described in Supplementary Information. Oligonucleotides are listed in Supplementary Data 6.

Q detection in tRNA. Overnight bacterial cultures were diluted $1 / 100$-fold into 5 $\mathrm{mL}$ of LB supplemented with $0.4 \%$ arabinose and $100 \mu \mathrm{g} / \mathrm{mL}$ ampicillin and grown for $2 \mathrm{~h}$ at $37^{\circ} \mathrm{C}$. Cells were harvested by centrifugation at $16,000 \times \mathrm{g}$ for $1 \mathrm{~min}$ at $4^{\circ}$ C. Cell pellets were immediately resuspended in $1 \mathrm{~mL}$ of Trizol (Life Technologies, Carlsbad, CA). Small RNAs were extracted using the PureLink ${ }^{\mathrm{TM}}$ miRNA Isolation Kit from Invitrogen (Carlsbad, CA) according to the manufacturer's protocol. Purified RNAs were eluted in $50 \mu \mathrm{L}$ of RNase-free water, and tRNA concentrations were measured with a NanoDrop ${ }^{\circledR}$ ND-1000 Spectrophotometer (Thermo Fisher Scientific, Waltham, MA). Then 200 ng of RNA was migrated in a $10 \%$ acrylamide/ bisacrylamide (29:1), Tris-EDTA acetate (TAE) $1 \times$, Urea $8 \mathrm{M}$ supplemented with 5 $\mu \mathrm{g} / \mathrm{mL} 3$-(acrylamido)-phenylboronic acid, as described in detail previously ${ }^{27}$. The migrated samples were transferred onto a Biodyne ${ }^{\mathrm{TM}}$ B Nylon membrane $(0.45$ $\mu \mathrm{m}$, Thermo Scientific, Rockford, IL). tRNA samples were detected using a (5'biotin-CCCTCGGTGACAGGCAGG-3') probe that anneals with tRNA $_{\text {Asp }}$ (GUC) at a final concentration of $0.3 \mu \mathrm{M}$ and the Chemiluminescent Nucleic Acid Detection Module Kit (Thermo Scientific, Rockford, IL), except that the first blocking buffer was changed to the DIG Easy Hyp buffer (Roche, Mannheim, Germany).

Restriction assay for deazapurine presence in plasmid DNA. E. coli strains containing different variations of pBAD24 and pBAD33 (with or without $d p d A$ or gat-queC from Enterobacteria phage $9 \mathrm{~g}$, see Supplementary Information) were grown overnight in LB supplemented with ampiciline $100 \mu \mathrm{g} / \mathrm{mL}$, chloramphenicol $20 \mu \mathrm{g} / \mathrm{mL}$ and $0.2 \%$ glucose at $37^{\circ} \mathrm{C}$. Each strain was diluted 100 -fold in LB supplemented with ampiciline $100 \mu \mathrm{g} / \mathrm{mL}$, chloramphenicol $20 \mu \mathrm{g} / \mathrm{mL}$ and $0.4 \%$ arabinose and grown for $6 \mathrm{~h}$ at $37^{\circ} \mathrm{C}$. Plasmids were extracted using the Qiagen QIAprep Spin Miniprep Kit, and 500 ng of plasmid was digested by EcoRI-HF (New England Biolabs, Ipswich MA) for $1 \mathrm{~h}$ at $37^{\circ} \mathrm{C}$ in $20 \mu \mathrm{L}$ of CutSmart buffer. The enzyme was inactivated by 20 -min incubation at $80^{\circ} \mathrm{C}$. The samples were run on a $0.5 \%$ agarose gel and TAE $1 \times$. The gel was then stained with $0.5 \mu \mathrm{g} / \mathrm{mL}$ ethidium bromide for $30 \mathrm{~min}$, washed 3 times for $15 \mathrm{~min}$ in water, and visualized with the Azur Biosystem c200 Gel Doc system (Thermo Fisher Scientific, Waltham, MA, USA).

Search for phage encoding $\mathbf{Q}$ and $\mathbf{G}^{+}$biosynthesis proteins. The Viruses $\mathrm{nr}$ database from NCBI was queried by three iterations of PSI-BLAST ${ }^{53}$, with the default set-up as previously suggested ${ }^{54}$, using the proteins referenced in Supplementary Table 1 known to be involved in $\mathrm{Q}$ or $\mathrm{G}^{+}$biosynthesis, as well as DpdA from Enterobacteria phage 9g, predicted to be involved in the modification of phage DNA, and another DpdA2 from Vibrio phage nt-1, part of a family identified in this study. The preQ $\mathrm{Q}_{0}$-specific transporter $\mathrm{YhhQ}^{33}$ was also added. For each virus identified with at least one of these genes, a reverse analysis was performed (phage genome against the protein list) to ensure that no protein was missed during the first analysis. The annotations for each identified ortholog were verified by HHpred ${ }^{55}$.

SSN generation. For each protein family (FolE, QueD, QueE, QueC/Gat-QueC, QueF/QueF-L, ArcS, and TGT), a representative set was imported from the OMA database $^{56}$. For the DpdA from bacteria, the protein sequences were imported from the genomes identified previously ${ }^{11}$ through PubSEED ${ }^{42}$. To generate the protein network, the sequences in fasta format were uploaded and analyzed online by the EFI-EST tool ${ }^{37}$. Each network was analyzed using the Cytoscape program ${ }^{57}$, and each family was clustered using the alignment score thresholds indicated in Fig. 3 and Supplementary Fig. 2.

Identification of the host and their gene content. The Virus-Host $\mathrm{DB}^{41}$ was used to obtain the host information for each phage identified in this study. For phages not referenced in this database, a manual investigation coupling RefSeq ${ }^{39}$ and the literature was performed (indicated as "manual" in the evidence line of Supplementary Data 3). Each host identified was queried in the Globi database ${ }^{40}$, and if they were identified as pathogens, the host was entered in the "Pathogen Of" column of Supplementary Data 3. The same analysis was performed for all the dsDNA phages of the Virus-Host DB, as only these phages were returned in our analysis (data not shown). A list of genomes was created on PubSEED ${ }^{42}$ from the identified hosts, and a spreadsheet was created. Proteins from Supplementary Table 1 were used to identify the correct annotation for each column of the spreadsheet. The results were collected and are shown in Supplementary Data 3.

Purification of phage and plasmid DNA. The purification of each phage DNA in this study was performed specifically for each phage and is described in Supplementary Information.

Mass spectrometric analysis. DNA analysis was performed as previously described with several modifications ${ }^{11}$. Purified DNA $(20 \mu \mathrm{g})$ was hydrolyzed in 10 $\mathrm{mM}$ Tris- $\mathrm{HCl}$ ( $\mathrm{pH} 7.9$ ) with $1 \mathrm{mM} \mathrm{MgCl}_{2}$ with benzonase (20 U), DNase I (4 U), calf intestine phosphatase $(17 \mathrm{U})$, and phosphodiesterase $(0.2 \mathrm{U})$ for $16 \mathrm{~h}$ at ambient temperature. Following passage through a $10-\mathrm{kDa}$ filter to remove proteins, the filtrate was lyophilized and resuspended to a final concentration of $0.2 \mu \mathrm{g} /$ $\mu \mathrm{L}$ (based on initial DNA quantity).

Quantification of the modified 2'-deoxynucleosides (dADG, dQ, dPreQ ${ }_{0}$, $\mathrm{dPreQ}_{1}$, and $\left.\mathrm{dG}^{+}\right)$and the four canonical $2^{\prime}$-deoxyribonucleosides $(\mathrm{dA}, \mathrm{dT}, \mathrm{dG}$, and $\mathrm{dC}$ ) was achieved by LC-MS/MS and an in-line diode array detector (LC$\mathrm{DAD})$, respectively. Aliquots of hydrolyzed DNA were injected onto a Phenomenex Luna Omega Polar C18 column $(2.1 \times 100 \mathrm{~mm}, 1.6 \mu \mathrm{m}$ particle size $)$ equilibrated with $98 \%$ solvent $\mathrm{A}(0.1 \% \mathrm{v} / \mathrm{v}$ formic acid in water) and $2 \%$ solvent $\mathrm{B}$ $(0.1 \% \mathrm{v} / \mathrm{v}$ formic acid in acetonitrile $)$ at a flow rate of $0.25 \mathrm{~mL} / \mathrm{min}$ and eluted with the following solvent gradient: $12 \%$ B for $10 \mathrm{~min}, 1 \mathrm{~min}$ ramp to $100 \% \mathrm{~B}$ for 10 $\mathrm{min}, 1 \mathrm{~min}$ ramp to $2 \% \mathrm{~B}$ for $10 \mathrm{~min}$. The high-performance liquid chromatographic column was coupled to an Agilent 1290 Infinity DAD and an Agilent 6490 triple quadruple mass spectrometer (Agilent, Santa Clara, CA). The column was kept at $40^{\circ} \mathrm{C}$, and the autosampler was cooled at $4{ }^{\circ} \mathrm{C}$. The ultraviolet wavelength of the DAD was set at $260 \mathrm{~nm}$ and the electrospray ionization of the mass spectrometer was performed in positive ion mode with the following source parameters: drying gas temperature, $200^{\circ} \mathrm{C}$ with a flow of $14 \mathrm{~L} / \mathrm{min}$; nebulizer gas 
pressure, $30 \mathrm{psi}$; sheath gas temperature, $400^{\circ} \mathrm{C}$ with a flow of $11 \mathrm{~L} / \mathrm{min}$; capillary voltage, $3,000 \mathrm{~V}$; and nozzle voltage, $800 \mathrm{~V}$. Compounds were quantified in multiple reaction monitoring mode with the following $\mathrm{m} / \mathrm{z}$ transitions: $310.1 \rightarrow$ $194.1,310.1 \rightarrow 177.1$, 310.1 $\rightarrow 293.1$ for dADG; $394.1 \rightarrow 163.1$, 394.1 $\rightarrow 146.1$ $394.1 \rightarrow 121.1$ for dQ; $292.1 \rightarrow 176.1,176.1 \rightarrow 159.1,176.1 \rightarrow 52.1$ for $\mathrm{dPreQ}$; $296.1 \rightarrow 163.1,296.1 \rightarrow 121.1,296.1 \rightarrow 279.1$ for $\mathrm{dPreQ}_{1}$; and $309.1 \rightarrow 193.1$, $309.1 \rightarrow 176.1$, $309.1 \rightarrow 159.1$ for $\mathrm{dG}^{+}$. External calibration curves were used to quantify the modified canonical 2 -deoxynucleosides. Calibration curves were constructed from replicate measurements of eight concentrations of each standard. A linear regression with $r^{2}>0.995$ was obtained in all relevant ranges. The limit of detection, defined by a signal-to-noise ratio $\geq 3$, ranged from 0.1 to $1 \mathrm{fmol}$ for the modified 2'-deoxynucleosides. Data acquisition and processing were performed using the MassHunter software (Agilent, Santa Clara, CA).

Phage genome editing using CRISPR-Cas9. Escherichia phage CAjan was genetically engineered as previously described ${ }^{58}$ and as summarized in Supplementary Fig. 2A. Briefly, E. coli MG1655 was transformed with two plasmids, pL2Cas9_dpdA $\Delta$ (see Supplementary Information for detailed construction method), which contained a spacer (5'-TGCGGTCAAGCCAAGTCT-

TAAGCGTGTCCG-3') targeting the $d p d A$ gene of Escherichia phage CAjan, and pNZ123_dpdA $\Delta$ (see Supplementary Information for detailed construction method), which carried a homologous repair template with a partially deleted, nonfunctional allele of the $d p d A$ gene (del29212-29521). Phage engineering was accomplished by infecting the modified host with WT Escherichia phage CAjan and isolating the resulting phage mutants. The infection step was repeated twice, and the resulting mutants were verified by PCR and whole-genome sequencing as described elsewhere ${ }^{59}$.

Restriction assay of phage DNA. A total of $250 \mathrm{ng}$ of phage DNA was digested by the enzymes (New England Biolabs) described in Fig. 6 for $1 \mathrm{~h}$ at $37^{\circ} \mathrm{C}$ in $20 \mu \mathrm{L}$ of CutSmart or 3.1 Buffer solution, according to the manufacturer's instructions. The enzymes were inactivated by incubation at $80^{\circ} \mathrm{C}$ for $20 \mathrm{~min}$. The samples were run on a $0.7 \%$ agarose gel and TAE $1 \times$. The gel was then stained for $30 \mathrm{~min}$ in $0.5 \mu \mathrm{g} /$ $\mathrm{mL}$ ethidium bromide, washed 3 times for $15 \mathrm{~min}$ in water, and visualized with the Azur Biosystem c200 Gel Doc system.

Reporting summary. Further information on research design is available in the Nature Research Reporting Summary linked to this article.

\section{Data availability}

Data supporting the findings of this work are available within the paper and its Supplementary Information files. A reporting summary for this article is available as a Supplementary Information file. The datasets generated and analyzed during the current study are available in Supplementary Information or from the corresponding author upon request. The source data are provided as a Source Data file.

Received: 1 May 2019; Accepted: 4 November 2019;

Published online: 29 November 2019

\section{References}

1. Chopin, M. C., Chopin, A. \& Bidnenko, E. Phage abortive infection in lactococci: variations on a theme. Curr. Opin. Microbiol. 8, 473-479 (2005).

2. Labrie, S. J., Samson, J. E. \& Moineau, S. Bacteriophage resistance mechanisms. Nat. Rev. Microbiol. 8, 317-327 (2010).

3. Golais, F., Hollý, J. \& Vítkovská, J. Coevolution of bacteria and their viruses. Folia Microbiol. (Praha) 58, 177-186 (2013).

4. Makarova, K. S. et al. An updated evolutionary classification of CRISPR-Cas systems. Nat. Rev. Microbiol. 13, 722-736 (2015).

5. Ershova, A. S., Rusinov, I. S., Spirin, S. A., Karyagina, A. S. \& Alexeevski, A. V. Role of restriction-modification systems in prokaryotic evolution and ecology. Biochemistry (Mosc.) 80, 1373-1386 (2015).

6. Samson, J. E., Magadán, A. H., Sabri, M. \& Moineau, S. Revenge of the phages: defeating bacterial defences. Nat. Rev. Microbiol. 11, 675-687 (2013).

7. Borges, A. L., Davidson, A. R. \& Bondy-Denomy, J. The discovery, mechanisms, and evolutionary impact of anti-CRISPRs. Annu. Rev. Virol. 4, 37-59 (2017)

8. Pawluk, A., Davidson, A. R. \& Maxwell, K. L. Anti-CRISPR: discovery, mechanism and function. Nat. Rev. Microbiol. 16, 12-17 (2018).

9. Bryson, A. L. et al. Covalent modification of bacteriophage T4 DNA inhibits CRISPR-Cas9. MBio 6, e00648 (2015).
10. Flodman, K. et al. Type II restriction of bacteriophage DNA with 5hmdUderived base modifications. Front. Microbiol. 10, 1-13 (2019).

11. Thiaville, J. J. et al. Novel genomic island modifies DNA with 7-deazaguanine derivatives. Proc. Natl Acad. Sci. USA 113, E1452-E1459 (2016).

12. Reader, J. S., Metzgar, D., Schimmel, P. \& De Crécy-Lagard, V. Identification of four genes necessary for biosynthesis of the modified nucleoside queuosine. J. Biol. Chem. 279, 6280-6285 (2004).

13. Phillips, G. et al. Biosynthesis of 7-deazaguanosine-modified tRNA nucleosides: a new role for GTP cyclohydrolase I. J. Bacteriol. 190, 7876-7884 (2008).

14. McCarty, R. M. \& Bandarian, V. Biosynthesis of pyrrolopyrimidines. Bioorg. Chem. 43, 15-25 (2012).

15. Phillips, G. et al. Discovery and characterization of an amidinotransferase involved in the modification of archaeal tRNA. J. Biol. Chem. 285, 12706-12713 (2010).

16. Phillips, G. et al. Diversity of archaeosine synthesis in Crenarchaeota. ACS Chem. Biol. 7, 300-305 (2012).

17. Bon Ramos, A., Bao, L., Turner, B., de Crécy-Lagard, V. \& Iwata-Reuyl, D. QueF-like, a non-homologous Archaeosine synthase from the Crenarchaeota. Biomolecules 7, 1-14 (2017).

18. Van Lanen, S. G. et al. From cyclohydrolase to oxidoreductase: discovery of nitrile reductase activity in a common fold. Proc. Natl Acad. Sci. USA 102, 4264-4269 (2005)

19. Stengl, B., Reuter, K. \& Klebe, G. Mechanism and substrate specificity of tRNA-guanine transglycosylases (TGTs): tRNA-modifying enzymes from the three different kingdoms of life share a common catalytic mechanism. ChemBioChem 6, 1926-1939 (2005).

20. Van Lanen, S. G. \& Iwata-Reuyl, D. Kinetic mechanism of the tRNAmodifying enzyme S-adenosylmethionine:tRNA ribosyltransferase-isomerase (QueA). Biochemistry 42, 5312-5320 (2003).

21. Miles, Z. D., McCarty, R. M., Molnar, G. \& Bandarian, V. Discovery of epoxyqueuosine (oQ) reductase reveals parallels between halorespiration and tRNA modification. Proc. Natl Acad. Sci. USA 108, 7368-7372 (2011).

22. Zallot, R. et al. Identification of a novel epoxyqueuosine reductase family by comparative genomics. ACS Chem. Biol. 12, 844-851 (2017).

23. Carstens, A. B., Kot, W. \& Hansen, L. H. Complete genome sequences of four novel Escherichia coli bacteriophages belonging to new phage groups. Genome Announc. 3, e00741-15 (2015).

24. Sabri, M. et al. Genome annotation and intraviral interactome for the streptococcus pneumoniae virulent phage Dp-1. J. Bacteriol. 193, 551-562 (2011).

25. Kot, W. et al. Complete genome sequence of Streptococcus pneumoniae virulent phage MS1. Genome Announc. 5, 9-10 (2017).

26. Pedulla, M. L. et al. Origins of highly mosaic mycobacteriophage genomes. Cell 113, 171-182 (2003).

27. Yuan, Y. et al. Identification of the minimal bacterial $2^{\prime}$-deoxy-7-amido-7deazaguanine synthesis machinery. Mol. Microbiol. 110, 469-483 (2018).

28. Kulikov, E. et al. Genomic sequencing and biological characteristics of a novel Escherichia coli bacteriophage $9 \mathrm{~g}$, a putative representative of a new Siphoviridae genus. Viruses 6, 5077-5092 (2014).

29. Tsai, R., Corrêa, I. R., Xu, M. Y. \& Xu, S. Y. Restriction and modification of deoxyarchaeosine (dG+)-containing phage 9 g DNA. Sci. Rep. 7, 1-13 (2017).

30. Mačková, M., Boháčová, S., Perlíková, P., Poštová Slavětínská, L. \& Hocek, M Polymerase synthesis and restriction enzyme cleavage of DNA containing 7 substituted 7-deazaguanine nucleobases. ChemBioChem 16, 2225-2236 (2015).

31. Hutinet, G., Swarjo, M. A. \& de Crécy-Lagard, V. Deazaguanine derivatives, examples of crosstalk between RNA and DNA modification pathways. RNA Biol. 14, 1175-1184 (2017).

32. Hanson, A. D. \& Gregory, J. F. Synthesis and turnover of folates in plants Curr. Opin. Plant Biol. 5, 244-249 (2002).

33. Zallot, R., Yuan, Y. \& De Crecy-Lagard, V. The Escherichia coli COG1738 member YhhQ is involved in 7-cyanodeazaguanine (preQ0) transport. Biomolecules 7, 1-13 (2017).

34. Russell, D. A. \& Hatfull, G. F. PhagesDB: the actinobacteriophage database. Bioinformatics 33, 784-786 (2017)

35. Tuorto, F. et al. Queuosine-modified tRNAs confer nutritional control of protein translation. EMBO J. 37, e99777 (2018).

36. Crippen, C. S. et al. Two subfamilies of Campylobacter jejuni bacteriophages replace genomic deoxyguanosine with alternative nucleobases. J. Virol. https:// doi.org/10.1128/JVI.01111-19 (2019).

37. Cicmil, N. \& Huang, R. H. Crystal structure of QueC from Bacillus subtilis: an enzyme involved in preQ1biosynthesis. Proteins Struct. Funct. Genet. 72, 1084-1088 (2008)

38. Gerlt, J. A. et al. Enzyme function initiative-enzyme similarity tool (EFI-EST): a web tool for generating protein sequence similarity networks. Biochim. Biophys. Acta 1854, 1019-1037 (2015). 
39. O’Leary, N. A. et al. Reference sequence (RefSeq) database at NCBI: current status, taxonomic expansion, and functional annotation. Nucleic Acids Res. 44, D733-D745 (2016)

40. Poelen, J. H., Simons, J. D. \& Mungall, C. J. Global biotic interactions: an open infrastructure to share and analyze species-interaction datasets. Ecol. Informatics 24, 148-159 (2014).

41. Mihara, T. et al. Linking virus genomes with host taxonomy. Viruses 8, 10-15 (2016).

42. Overbeek, R. et al. The subsystems approach to genome annotation and its use in the project to annotate 1000 genomes. Nucleic Acids Res. 33, 5691-5702 (2005).

43. Carstens, A. B., Kot, W., Lametsch, R., Neve, H. \& Hansen, L. H. Characterisation of a novel enterobacteria phage, CAjan, isolated from rat faeces. Arch. Virol. 161, 2219-2226 (2016).

44. Lemay, M.-L., Renaud, A., Rousseau, G. \& Moineau, S. Targeted genome editing of virulent phages using CRISPR-Cas9. Bio-protocol 7, 1-19 (2018).

45. Vourvahis, M. et al. Excretion and Metabolism of lersivirine (5-\{[3,5-Diethyl1-(2-hydroxyethyl)(3,5-14C2)-1H-pyrazol-4-yl] oxy\}benzene-1,3dicarbonitrile), a next-generation non-nucleoside reverse transcriptase inhibitor, after administration of [14C]Lersivirine to healthy volunteers. Drug Metab. Dispos. 38, 789-800 (2010).

46. Mei, X. et al. Crystal structure of the archaeosine synthase QueF-like-insights into amidino transfer and tRNA recognition by the tunnel fold. Proteins 165, 255-269 (2016).

47. Weigele, P. \& Raleigh, E. A. Biosynthesis and function of modified bases in bacteria and their viruses. Chem. Rev. 116, 12655-12687 (2016).

48. Lee, Y.-J. et al. Identification and biosynthesis of thymidine hypermodifications in the genomic DNA of widespread bacterial viruses. Proc. Natl Acad. Sci. USA 115, E3116-E3125 (2018).

49. Nechaev, S. \& Severinov, K. Bacteriophage-induced modifications of host RNA polymerase. Annu. Rev. Microbiol. 57, 301-322 (2004).

50. Feklístov, A., Sharon, B. D., Darst, S. A. \& Gross, C. A. Bacterial sigma factors: a historical, structural, and genomic perspective. Annu. Rev. Microbiol. 68, 357-376 (2014).

51. Yang, H. et al. Transcription regulation mechanisms of bacteriophages. Bioengineered 5, 300-304 (2014).

52. Gleditzsch, D. et al. PAM identification by CRISPR-Cas effector complexes: diversified mechanisms and structures. RNA Biol. 16, 504-517 (2019).

53. Altschul, S. F. et al. Gapped BLAST and PSI-BLAST: a new generation of protein database search programs. Nucleic Acids Res. 25, 3389-3402 (1997).

54. Lopes, A., Amarir-Bouhram, J., Faure, G., Petit, M. A. \& Guerois, R. Detection of novel recombinases in bacteriophage genomes unveils Rad52, Rad51 and Gp2.5 remote homologs. Nucleic Acids Res. 38, 3952-3962 (2010).

55. Söding, J. Protein homology detection by HMM-HMM comparison. Bioinformatics 21, 951-960 (2005).

56. Altenhoff, A. M. et al. The OMA orthology database in 2018: retrieving evolutionary relationships among all domains of life through richer web and programmatic interfaces. Nucleic Acids Res. 46, D477-D485 (2018).

57. Shannon, P. et al. Cytoscape: a software environment for integrated models of biomolecular interaction networks. Genome Res. 13, 2498-2504 (2003).

58. Lemay, M. L., Tremblay, D. M. \& Moineau, S. Genome engineering of virulent lactococcal phages using CRISPR-Cas9. ACS Synth. Biol. 6 1351-1358 (2017).

59. Kot, W., Vogensen, F. K., Sørensen, S. J. \& Hansen, L. H. DPS-a rapid method for genome sequencing of DNA-containing bacteriophages directly from a single plaque. J. Virol. Methods 196, 152-156 (2014).

\section{Acknowledgements}

This work was funded in part by the National Institutes of Health (grant GM70641 to V.d.C.-L. and P.C.D.), the Human Frontier Science Program (grant RGP0024 to L.H., V.d.C.-L., and S.M.), and the Villum Experiment (grant 17595 to W.K.). We thank the 2009 and 2016 MIT students of the 7.396 independent activity period class who isolated the Pseudomonas phage Quinobequin P09; Cameron Haase-Pettingell for assistance with electron microscopy; Dennis Bamford for Halovirus HVTV-1 and its host Haloarcula vallismortis and Audrey Jonas for preparations of phages Orion and Rosebush; Rémi Zallot for SSN tutorials and Gabriella Phillips for pCH111 plasmid construction; and Marie-Laurence Lemay for her help with the genome editing of Escherichia phage CAjan. We are grateful to Marie-Agnès Petit for critical reading of the manuscript and Christine Szymanski for sharing information on Campylobacter phages. S.M. holds the Tier 1 Canada Research Chair in Bacteriophages.

\section{Author contributions}

G.H., W.K., L.C., R.H., S.B., S.G., R.N., A.B.C., C.F.L., M.S., Y.J.L., and P.W. performed the experimental work. G.H., W.K., and L.C. contributed to the manuscript preparation. D.T., D.J.-S., S.M., G.F.H., P.C.D., L.H.H., and V.d.C.-L. contributed their expertise and supervision to the work. G.H. and V.d.C.-L. conceived the idea and supervised the entire project. G.H., W.K., L.C., M.S., P.W., and V.d.C.-L. wrote the manuscript.

\section{Competing interests}

The authors declare no competing interests.

\section{Additional information}

Supplementary information is available for this paper at https://doi.org/10.1038/s41467 019-13384-y.

Correspondence and requests for materials should be addressed to G.H. or V.dC.-L.

Peer review information Nature Communications thanks Lawrence Sowers, Shuangyong Xu2 and the other, anonymous, reviewer(s) for their contribution to the peer review of this work. Peer reviewer reports are available.

Reprints and permission information is available at http://www.nature.com/reprints Publisher's note Springer Nature remains neutral with regard to jurisdictional claims in published maps and institutional affiliations.

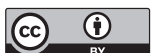

Open Access This article is licensed under a Creative Commons Attribution 4.0 International License, which permits use, sharing, adaptation, distribution and reproduction in any medium or format, as long as you give appropriate credit to the original author(s) and the source, provide a link to the Creative Commons license, and indicate if changes were made. The images or other third party material in this article are included in the article's Creative Commons license, unless indicated otherwise in a credit line to the material. If material is not included in the article's Creative Commons license and your intended use is not permitted by statutory regulation or exceeds the permitted use, you will need to obtain permission directly from the copyright holder. To view a copy of this license, visit http://creativecommons.org/ licenses/by/4.0/.

This is a U.S. government work and not under copyright protection in the U.S.; foreign copyright protection may apply 2019 